Groups Geom. Dyn. 8 (2014), 355-374

DOI $10.4171 / \mathrm{GGD} / 229$
Groups, Geometry, and Dynamics

(C) European Mathematical Society

\title{
A note on trace fields of complex hyperbolic groups
}

\author{
Heleno Cunha and Nikolay Gusevskii
}

\begin{abstract}
We show that if $\Gamma$ is an irreducible subgroup of $\mathrm{SU}(2,1)$, then $\Gamma$ contains a loxodromic element $A$. If $A$ has eigenvalues $\lambda_{1}=\lambda e^{i \varphi}, \lambda_{2}=e^{-2 i \varphi}, \lambda_{3}=\lambda^{-1} e^{i \varphi}$, we prove that $\Gamma$ is conjugate in $\mathrm{SU}(2,1)$ to a subgroup of $\mathrm{SU}(2,1, \mathbb{Q}(\Gamma, \lambda))$, where $\mathbb{Q}(\Gamma, \lambda)$ is the field generated by the trace field $\mathbb{Q}(\Gamma)$ of $\Gamma$ and $\lambda$. It follows from this that if $\Gamma$ is an irreducible subgroup of $\operatorname{SU}(2,1)$ such that the trace field $\mathbb{Q}(\Gamma)$ is real, then $\Gamma$ is conjugate in $\operatorname{SU}(2,1)$ to a subgroup of $\mathrm{SO}(2,1)$. As a geometric application of the above, we get that if $G$ is an irreducible discrete subgroup of $\mathrm{PU}(2,1)$, then $G$ is an $\mathbb{R}$-Fuchsian subgroup of $\mathrm{PU}(2,1)$ if and only if the invariant trace field $k(G)$ of $G$ is real.
\end{abstract}

Mathematics Subject Classification (2010). 32H20, 20H10, 22E40, 57S30, 32G07, 32C16.

Keywords. Complex hyperbolic groups, trace fields.

\section{Introduction}

Arithmetic methods are a powerful tool in the study of Kleinian groups, discrete subgroups of PSL $(2, \mathbb{C})$, especially of finite-covolume discrete groups, as was demonstrated in [21], see also an extensive bibliography there. A central theme in this theory is to understand the structure of the invariant trace field and the invariant (quaternion) algebra associated to a Kleinian group. In the case of complex hyperbolic geometry, that is, in the case of subgroups of $\mathrm{PU}(n, 1)(\mathrm{SU}(n, 1))$ little known about these objects, see for instance [23], where the study of the invariant trace fields and the invariant algebras associated to subgroups of $\mathrm{SU}(n, 1)$ was initiated. In particular, in this work the invariant trace field and the invariant algebra were introduced for subgroups of $\mathrm{SU}(n, 1)$. An important problem here is to understand whether a subgroup of $\mathrm{SU}(n, 1)$ can be realized over the field generated by the eigenvalues of its elements. In this paper, we prove that any irreducible subgroup $\Gamma$ of $\mathrm{SU}(2,1)$ contains a loxodromic element $A$ and it can be realized over the field generated by the trace field of $\Gamma$ and the eigenvalues of $A$.

The main result of our paper is the following theorem:

Theorem A. Let $\Gamma$ be an irreducible subgroup of $\mathrm{SU}(2,1)$ and $A \in \Gamma$ be loxodromic with eigenvalues $\lambda_{1}=\lambda e^{i \varphi}, \lambda_{2}=e^{-2 i \varphi}, \lambda_{3}=\lambda^{-1} e^{i \varphi}$. Then $\Gamma$ is conjugate in 
$\mathrm{SU}(2,1)$ to a subgroup of $\mathrm{SU}(2,1, \mathbb{Q}(\Gamma, \lambda))$, where $\mathbb{Q}(\Gamma, \lambda)$ is the field generated by the trace field $\mathbb{Q}(\Gamma)$ of $\Gamma$ and $\lambda$.

As a corollary of this theorem, we get the following

Theorem B. Let $\Gamma$ be an irreducible subgroup of $\mathrm{SU}(2,1)$ such that $\mathbb{Q}(\Gamma)$ is a subset of $\mathbb{R}$, then $\Gamma$ is conjugate in $\mathrm{SU}(2,1)$ to a subgroup of $\mathrm{SO}(2,1)$.

We would like to stress that in Theorem A and Theorem B we do not assume that the group $\Gamma$ is discrete.

Also, in this paper, we define an invariant trace field for subgroups of $\mathrm{PU}(2,1)$. Let $G$ be a subgroup of $\mathrm{PU}(2,1)$ and $\Gamma=\pi^{-1}(G)$, where $\pi: \operatorname{SU}(2,1) \rightarrow \mathrm{PU}(2,1)$ is a natural projection. Then the invariant trace field of $G$, denoted by $k(G)$, is defined to be the field $\mathbb{Q}\left(\Gamma^{3}\right)$, where $\Gamma^{3}=\left\langle\gamma^{3}: \gamma \in \Gamma\right\rangle$. It follows from [23] that the invariant trace field is an invariant of the commensurability class.

We say that a subgroup $G$ of $\mathrm{PU}(2,1)$ is an $\mathbb{R}$-subgroup if it leaves invariant a totally real geodesic 2-plane in $\boldsymbol{H}_{\mathbb{C}}^{2}$. A subgroup $G$ of PU $(2,1)$ is called $\mathbb{R}$-Fuchsian if it is a discrete $\mathbb{R}$-subgroup. A subgroup $G$ is a $\mathbb{C}$-subgroup if it leaves invariant a complex geodesic in $\boldsymbol{H}_{\mathbb{C}}^{2}$. A subgroup $G$ of $\mathrm{PU}(2,1)$ is called $\mathbb{C}$-Fuchsian if it is a discrete $\mathbb{C}$-subgroup.

By applying Theorem B, we get the following characterization of discrete nonelementary $\mathbb{R}$-subgroups of $\mathrm{PU}(2,1)$.

Theorem C. Let $G$ be an irreducible discrete subgroup of $\mathrm{PU}(2,1)$. Then $G$ is an $\mathbb{R}-$ Fuchsian if and only if the invariant trace field $k(G)$ of $G$ is real.

This implies, in particular, that if $G$ is an irreducible discrete subgroup of $\mathrm{PU}(2,1)$ whose invariant trace field is real, then the invariant algebra associated to $G$ is of dimension nine over $k(G)$. On the other hand, if $G$ is a non-elementary $\mathbb{C}$-subgroup of $\mathrm{PU}(2,1))(G$ is reducible in this case) with real invariant trace field, then the invariant algebra associated to $G$ is of dimension four.

As a corollary of Theorem $\mathrm{C}$, we have the following.

Theorem D. Let $G$ be a discrete non-elementary subgroup of $\mathrm{PU}(2,1)$ such that the invariant trace field $k(G)$ of $G$ is real. Then $G$ is either $\mathbb{R}$-Fuchsian or $\mathbb{C}$-Fuchsian.

We remark that Theorem $\mathrm{D}$ can be considered as a complex hyperbolic analog of a classical result due to B.Maskit, see Theorem G.18 in [22] and Corollary 3.2.5 in [21]. Finally, we would like to mention that some related questions were considered in [3], [14], [15], [24], [27], [28], [29], [30].

The article is organized as follows. In Section 1, we review some basic facts in complex hyperbolic geometry. In Section 2, we prove our main results. 


\section{Complex hyperbolic plane and its isometry group}

Let $V$ be a 3-dimensional $\mathbb{C}$-vector space equipped with a Hermitian form $\langle-,-\rangle$ of signature $(2,1)$. We denote by $\mathbb{P}(V)$ the complex projectivization of $V$ and by $\mathbb{P}: V \backslash\{0\} \rightarrow \mathbb{P}(V)$ a natural projection.

Let $V_{-}, V_{0}, V_{+}$be the subsets of $V \backslash\{0\}$ consisting of vectors where $\langle v, v\rangle$ is negative, zero, or positive respectively. Vectors in $V_{0}$ are called null or isotropic, vectors in $V_{-}$are called negative, and vectors in $V_{+}$are called positive. Their projections to $\mathbb{P}(V)$ are called isotropic, negative, and positive points respectively.

The projective model of the complex hyperbolic plane $\boldsymbol{H}_{\mathbb{C}}^{2}$ is the set of negative points in $\mathbb{P}(V)$, that is, $\boldsymbol{H}_{\mathbb{C}}^{2}=\mathbb{P}\left(V_{-}\right)$. The boundary $\partial \boldsymbol{H}_{\mathbb{C}}^{2}=\mathbb{P}\left(V_{0}\right)$ of $\boldsymbol{H}_{\mathbb{C}}^{2}$ is the 3 -sphere formed by all isotropic points.

The Hermitian form $\langle-,-\rangle$ defines a metric, the Bergman metric, on $\boldsymbol{H}_{\mathbb{C}}^{2}$, see [16]. Let $\mathrm{U}(V)$ be the unitary group corresponding to this Hermitian form. Then the holomorphic isometry group of $\boldsymbol{H}_{\mathbb{C}}^{2}$ is the projective unitary group $\mathrm{PU}(V)$, and the full isometry group of $\boldsymbol{H}_{\mathbb{C}}^{2}$ is generated by $\mathrm{PU}(V)$ and complex conjugation. We denote by $\mathrm{SU}(V)$ the subgroup of linear transformations in $\mathrm{U}(V)$ with determinant 1 .

For our purposes it is convenient to work with a basis $e=\left\{e_{1}, e_{2}, e_{3}\right\}$ in $V$ which has the following properties:

$$
\begin{array}{ll}
\left\langle e_{1}, e_{1}\right\rangle=0, & \left\langle e_{2}, e_{2}\right\rangle=1, \quad\left\langle e_{3}, e_{3}\right\rangle=0, \\
\left\langle e_{1}, e_{2}\right\rangle=0, & \left\langle e_{2}, e_{3}\right\rangle=0, \quad\left\langle e_{1}, e_{3}\right\rangle=1 .
\end{array}
$$

So, in this basis $e_{1}$ and $e_{3}$ are isotropic and $e_{2}$ is positive. In what follows, we denote by $\mathbb{C}^{2,1}$ the vector space $V$ equipped with this basis. If the vectors $z=\left(z_{1}, z_{2}, z_{3}\right)^{\top}$ and $w=\left(w_{1}, w_{2}, w_{3}\right)^{\top}$ in $\mathbb{C}^{2,1}$ are given by their coordinates in $e=\left\{e_{1}, e_{2}, e_{3}\right\}$, then the Hermitian product $\langle v, w\rangle$ is given by

$$
\langle v, w\rangle=z_{1} \bar{w}_{3}+z_{2} \bar{w}_{2}+z_{3} \bar{w}_{1} .
$$

The use of this basis simplifies essentially matrix computations and it was successfully applied in a series of works, see, for instance [6], [7], [8], [9], [10], [11], [12], [13], [18], [19], [29]. Throughout this paper we will use this basis.

Let $\mathrm{U}(2,1)$ and $\mathrm{SU}(2,1)$ denote the representations of $\mathrm{U}(V)$ and $\mathrm{SU}(V)$ in the basis $e=\left\{e_{1}, e_{2}, e_{3}\right\}$.

If $A$ is an element of $\mathrm{SU}(2,1)$, then the matrix $A$ is defined by the following simple conditions:

$$
\begin{aligned}
& \left\langle v_{1}, v_{1}\right\rangle=0, \quad\left\langle v_{2}, v_{2}\right\rangle=1, \quad\left\langle v_{3}, v_{3}\right\rangle=0 \\
& \left\langle v_{1}, v_{2}\right\rangle=0, \quad\left\langle v_{2}, v_{3}\right\rangle=0, \quad\left\langle v_{1}, v_{3}\right\rangle=1,
\end{aligned}
$$

where $v_{1}, v_{2}, v_{3}$ denote the vectors defined by the rows of $A$.

Also, we have the following useful formula for the inverse of $A \in \mathrm{SU}(2,1)$ :

$$
A=\left[\begin{array}{lll}
a_{11} & a_{12} & a_{13} \\
a_{21} & a_{22} & a_{23} \\
a_{31} & a_{32} & a_{33}
\end{array}\right], \quad A^{-1}=\left[\begin{array}{lll}
\bar{a}_{33} & \bar{a}_{23} & \bar{a}_{13} \\
\bar{a}_{32} & \bar{a}_{22} & \bar{a}_{12} \\
\bar{a}_{31} & \bar{a}_{21} & \bar{a}_{11}
\end{array}\right] .
$$


It is seen that $A^{-1}$ is the Hermitian anti-transpose of $A$.

The non-trivial elements of $\mathrm{PU}(2,1)$ fall into three general conjugacy types, depending on the number and location of their fixed points.

- Elliptic elements have a fixed point in $\boldsymbol{H}_{\mathbb{C}}^{2}$,

- Parabolic elements have a single fixed point on the boundary of $\boldsymbol{H}_{\mathbb{C}}^{2}$,

- Loxodromic elements have exactly two fixed points on the boundary of $\boldsymbol{H}_{\mathbb{C}}^{2}$.

This exhausts all possibilities, see [16] for details.

Let $\pi: \mathrm{SU}(2,1) \rightarrow \mathrm{PU}(2,1)$ be a natural projection. We call an element $A \in$ $\mathrm{SU}(2,1)$ loxodromic (parabolic, elliptic) if its projectivization $\pi(A)$ is loxodromic (parabolic, elliptic). For instance, any loxodromic element $A \in \mathrm{SU}(2,1)$ is conjugate in $\mathrm{SU}(2,1)$ to an element of the following form

$$
A=\left[\begin{array}{ccc}
\lambda_{1} & 0 & 0 \\
0 & \lambda_{2} & 0 \\
0 & 0 & \lambda_{3}
\end{array}\right],
$$

where $\lambda_{1}=\lambda e^{i \varphi}, \lambda_{2}=e^{-2 i \varphi}, \lambda_{3}=\lambda^{-1} e^{i \varphi}, \lambda>0, \lambda \neq 1, \varphi \in(-\pi, \pi]$.

A parabolic element $g \in \mathrm{PU}(2,1)$ is unipotent if it can be represented by a unipotent element of $\mathrm{SU}(2,1)$, that is, a matrix having 1 as its only eigenvalue. Otherwise, $g$ is ellipto-parabolic. In that case $g$ can be represented by an element of $\mathrm{SU}(2,1)$ having a repeated non-real eigenvalue of norm 1. Also, $g$ has a unique invariant complex geodesic, see below.

There are two types of totally geodesic submanifolds of $\boldsymbol{H}_{\mathbb{C}}^{2}$ of real dimension two:

- Complex geodesics (copies of $\boldsymbol{H}_{\mathbb{C}}^{1}$ ) have constant sectional curvature -1 .

- Totally real geodesic 2-planes (copies of $\boldsymbol{H}_{\mathbb{R}}^{2}$ ) have constant sectional curvature $-1 / 4$.

Any complex geodesic is the intersection of a complex projective line in $\mathbb{P}(V)$ with $\boldsymbol{H}_{\mathbb{C}}^{2}$, and it is uniquely defined by its polar point, which is positive [16]. We recall that a polar point to a complex projective line $c$ in $\mathbb{P}(V)$ is the projectivization of the Hermitian orthogonal complement in $V$ of $\mathbb{P}^{-1}(c)$.

Any totally real geodesic 2-plane is the intersection of a totally real projective plane in $\mathbb{P}(V)$ with $\boldsymbol{H}_{\mathbb{C}}^{2}$.

We recall that a subspace $S$ of $V_{\mathbb{R}}$, where $V_{\mathbb{R}}$ is the real vector space underlying $V$, is totally real if and only if $S$ and its image $\mathbb{J}(S)$ are orthogonal with respect to the Hermitian product $\langle-,-\rangle$, see [16]. It is easy to show that $S$ is totally real if and only if the Hermitian product $\langle v, u\rangle$ is real for all $v, u \in S$. An example of a totally real subspace is the $\mathbb{R}$-linear span of $e=\left\{e_{1}, e_{2}, e_{3}\right\}$, the basis considered above. We call this subspace $a$ canonical totally real subspace. The projectivization of this space is called a canonical totally real projective 2-plane. Any totally real 2-plane in $\mathbb{P}(V)$ is the image of the canonical totally real projective 2-plane under an element from the 
group $\mathrm{PU}(2,1)$. The stabilizer of the canonical totally real subspace in $\mathrm{SU}(2,1)$ can be canonically identified with the group $\mathrm{SO}(2,1)$.

A chain is the boundary of a complex geodesic. An $\mathbb{R}$-circle is the boundary of a totally real geodesic 2-plane, see [16].

Let $G$ be a subgroup of $\mathrm{PU}(2,1)$. We say that $G$ is a $\mathbb{C}$-subgroup if it leaves invariant a complex geodesic. $G$ is called an $\mathbb{R}$-subgroup if it leaves invariant a totally real geodesic 2-plane. A subgroup $\Gamma$ of $\mathrm{SU}(2,1)$ is called $\mathbb{C}$-subgroup if its projectivization is a $\mathbb{C}$-subgroup of $\mathrm{PU}(2,1)$. Similarly, a subgroup $\Gamma$ of $\mathrm{SU}(2,1)$ is called an $\mathbb{R}$-subgroup if its projectivization is an $\mathbb{R}$-subgroup of $\mathrm{PU}(2,1)$. We say that a subgroup $G$ of $\mathrm{PU}(2,1)$ is $\mathbb{C}$-Fuchsian if it is a discrete $\mathbb{C}$-subgroup, and $G$ is called $\mathbb{R}$-Fuchsian if it is a discrete $\mathbb{R}$-subgroup. Typical examples of $\mathbb{C}$-subgroups of $\mathrm{SU}(2,1)$ are subgroups of $\mathrm{SU}(1,1)$ canonically embedded into $\mathrm{SU}(2,1)$, and typical examples of $\mathbb{R}$-subgroups of $\mathrm{SU}(2,1)$ are subgroups of $\mathrm{SO}(2,1)$ canonically embedded into $\mathrm{SU}(2,1)$.

Recall that a subgroup $\Gamma$ of $\mathrm{SU}(2,1)$ is called reducible if it has an invariant proper $\mathbb{C}$-subspace of $V$, and called irreducible otherwise. It is clear that a subgroup $\Gamma$ of $\mathrm{SU}(2,1)$ is irreducible if and only if $\Gamma$ has no invariant 1 -dimensional $\mathbb{C}$-subspaces of $V$.

A subgroup $G$ of $\mathrm{PU}(2,1)$ is called reducible if all elements of $G$ have a common fixed point in their action on the projective space $\mathbb{P}(V)$. Otherwise, $G$ is called irreducible.

A subgroup $G$ of $\mathrm{PU}(2,1)$ is called elementary if it has a finite orbit in its action on $\boldsymbol{H}_{\mathbb{C}}^{2} \cup \partial \boldsymbol{H}_{\mathbb{C}}^{2}$. Otherwise, $G$ is non-elementary. A subgroup $\Gamma$ of $\mathrm{SU}(2,1)$ is called elementary if its projectivization is elementary. Otherwise, $\Gamma$ is non-elementary.

Clearly, any $\mathbb{C}$-subgroup $G$ of $\mathrm{PU}(2,1)$ is reducible because the polar point to the invariant complex geodesic of $G$ is a common fixed point for all elements of $G$ in their action on $\mathbb{P}(V)$. Also, it is clear that a non-elementary $\mathbb{R}$-subgroup of $\mathrm{PU}(2,1)$ is irreducible.

\section{Trace fields of complex hyperbolic groups}

Let $\Gamma$ be a subgroup of $\mathrm{SU}(2,1)$. Then the trace field of $\Gamma$, denoted by $\mathbb{Q}(\operatorname{tr} \Gamma)$, is the field generated by the traces of all the elements of $\Gamma$ over the base field $\mathbb{Q}$ of rational numbers. For simplicity, we will denote the trace field of $\Gamma$ by $\mathbb{Q}(\Gamma)$. Of course, $\mathbb{Q}(\Gamma)$ is a conjugacy invariant. We remark that the trace field $\mathbb{Q}(\Gamma)$ is also invariant under complex conjugation, since for $B \in \mathrm{SU}(2,1)$ we have $\operatorname{tr}\left(B^{-1}\right)=\overline{\operatorname{tr}(B)}$.

If $\Gamma$ contains a loxodromic element $A$ with eigenvalues $\lambda_{1}=\lambda e^{i \varphi}, \lambda_{2}=e^{-i 2 \varphi}$, $\lambda_{3}=\lambda^{-1} e^{i \varphi}$, then $\mathbb{Q}(\Gamma, \lambda)$ denotes the field generated by $\mathbb{Q}(\Gamma)$ and $\lambda$. Also, for any field $k$ we denote by $\mathrm{SU}(2,1, k)$ the intersection of $\mathrm{SU}(2,1)$ with $\mathrm{M}(3, k)$. 
Lemma 2.1. Let $\Gamma=\langle A\rangle$ be the group generated by a loxodromic element $A \in$ $\mathrm{SU}(2,1)$ with eigenvalues $\lambda_{1}=\lambda e^{i \varphi}, \lambda_{2}=e^{-2 i \varphi}, \lambda_{3}=\lambda^{-1} e^{i \varphi}$. Then $e^{i \varphi} \in$ $\mathbb{Q}(\Gamma, \lambda)$.

Proof. Since $\mathbb{Q}(\Gamma)$ is invariant under complex conjugation, it follows that $\operatorname{Re}(\operatorname{tr}(A))$ and $|\operatorname{tr}(A)|^{2}$ are in $\mathbb{Q}(\Gamma)$. We have that

$$
\operatorname{tr}(A)=\lambda e^{i \varphi}+\lambda^{-1} e^{i \varphi}+e^{-i 2 \varphi}
$$

and

$$
\operatorname{tr}\left(A^{-1}\right)=\overline{\operatorname{tr}(A)}=\lambda e^{-i \varphi}+\lambda^{-1} e^{-i \varphi}+e^{i 2 \varphi} .
$$

A direct computation shows that

$$
\operatorname{Re}(\operatorname{tr}(A))=\cos 2 \varphi+\left(\lambda+\lambda^{-1}\right) \cos \varphi=2 \cos ^{2} \varphi+\left(\lambda+\lambda^{-1}\right) \cos \varphi-1
$$

and

$$
|\operatorname{tr}(A)|^{2}=\left(\lambda^{2}+\lambda^{-2}\right)+2\left(\lambda+\lambda^{-1}\right) \cos 3 \varphi+3 .
$$

Since $\lambda+\lambda^{-1} \neq 0$, the last formula implies that $\cos 3 \varphi \in \mathbb{Q}(\Gamma, \lambda)$.

Looking at these formulae, one could expect that $\cos \varphi$ is in a proper extension of $\mathbb{Q}(\Gamma, \lambda)$, but the following trick shows that, in fact, $\cos \varphi$ is in $\mathbb{Q}(\Gamma, \lambda)$.

It is easy to see that the following formula

$$
\left(4 \cos ^{2} \varphi+2 t \cos \varphi+t^{2}-3\right) \cos \varphi=4 \cos ^{3} \varphi-3 \cos \varphi+\left(2 \cos ^{2} \varphi+t \cos \varphi\right) t
$$

is true for all $t \in \mathbb{R}$ and for all $\varphi \in(-\pi, \pi]$.

Also, it is elementary to check that

$$
4 \cos ^{2} \varphi+2 t \cos \varphi+t^{2}-3 \neq 0
$$

for all $t>2$ and for all $\varphi \in(-\pi, \pi]$. This implies that for all $t>2$ and for all $\varphi \in(-\pi, \pi]$ we have that

$$
\cos \varphi=\frac{4 \cos ^{3} \varphi-3 \cos \varphi+\left(2 \cos ^{2} \varphi+t \cos \varphi\right) t}{4 \cos ^{2} \varphi+2 t \cos \varphi+t^{2}-3} .
$$

In particular, taking $t=\lambda+\lambda^{-1}$ and using the identity $\cos 3 \varphi=4 \cos ^{3} \varphi-3 \cos \varphi$, we get the formula

$$
\cos \varphi=\frac{\cos 3 \varphi+\left(2 \cos ^{2} \varphi+\left(\lambda+\lambda^{-1}\right) \cos \varphi\right)\left(\lambda+\lambda^{-1}\right)}{4 \cos ^{2} \varphi+2\left(\lambda+\lambda^{-1}\right) \cos \varphi+\left(\lambda+\lambda^{-1}\right)^{2}-3} .
$$

One verifies that this can be re-written as

$$
\cos \varphi=\frac{\cos 3 \varphi+(\operatorname{Re}(\operatorname{tr}(A))+1)\left(\lambda+\lambda^{-1}\right)}{2 \operatorname{Re}(\operatorname{tr}(A))+\left(\lambda+\lambda^{-1}\right)^{2}-1} .
$$


Therefore, the above implies that $\cos \varphi \in \mathbb{Q}(\Gamma, \lambda)$ for all $\varphi \in(-\pi, \pi]$.

Next, a direct computation shows that

$$
i \sin \varphi=\frac{\operatorname{tr}(A)-\overline{\operatorname{tr}(A)}}{2\left(\lambda+\lambda^{-1}+2 \cos \varphi\right)} .
$$

All the above implies that $e^{i \varphi} \in \mathbb{Q}(\Gamma, \lambda)$ for all $\varphi \in(-\pi, \pi]$.

Corollary 2.1. Let $\Gamma=\langle A\rangle$ be the group generated by a loxodromic element $A \in$ $\mathrm{SU}(2,1)$ with eigenvalues $\lambda_{1}=\lambda e^{i \varphi}, \lambda_{2}=e^{-i 2 \varphi}, \lambda_{3}=\lambda^{-1} e^{i \varphi}$. Then all these eigenvalues belong to $\mathbb{Q}(\Gamma, \lambda)$.

Lemma 2.2. Let $\Gamma$ be a subgroup of $\mathrm{SU}(2,1)$ containing a loxodromic element $A=\operatorname{diag}\left(\lambda_{1}, \lambda_{2}, \lambda_{3}\right)$, where $\lambda_{1}=\lambda e^{i \varphi}, \lambda_{2}=e^{-i 2 \varphi}, \lambda_{3}=\lambda^{-1} e^{i \varphi}$. Then for any element $B=\left(b_{i j}\right) \in \Gamma$, the diagonal elements of $B$ are in $\mathbb{Q}(\Gamma, \lambda)$.

Proof. We write

$$
A=\left[\begin{array}{ccc}
\lambda_{1} & 0 & 0 \\
0 & \lambda_{2} & 0 \\
0 & 0 & \lambda_{3}
\end{array}\right], \quad A^{-1}=\left[\begin{array}{ccc}
\bar{\lambda}_{3} & 0 & 0 \\
0 & \bar{\lambda}_{2} & 0 \\
0 & 0 & \bar{\lambda}_{1}
\end{array}\right], \quad B=\left[\begin{array}{lll}
b_{11} & b_{12} & b_{13} \\
b_{21} & b_{22} & b_{23} \\
b_{31} & b_{32} & b_{33}
\end{array}\right] .
$$

Then computations show that

$$
\begin{aligned}
\operatorname{tr}(B) & =b_{11}+b_{22}+b_{33}=t_{1}, \\
\operatorname{tr}(A B) & =\lambda_{1} b_{11}+\lambda_{2} b_{22}+\lambda_{3} b_{33}=t_{2}, \\
\operatorname{tr}\left(A^{-1} B\right) & =\bar{\lambda}_{3} b_{11}+\bar{\lambda}_{2} b_{22}+\bar{\lambda}_{1} b_{33}=t_{3} .
\end{aligned}
$$

Note that every $t_{i}$ lies in $\mathbb{Q}(\Gamma)$. We consider these equalities as a system of linear equations in three unknowns $b_{11}, b_{22}, b_{33}$. Let us show that the matrix $L$ of this system is non-singular. We write

$$
L=\left[\begin{array}{ccc}
1 & 1 & 1 \\
\lambda_{1} & \lambda_{2} & \lambda_{3} \\
\bar{\lambda}_{3} & \bar{\lambda}_{2} & \bar{\lambda}_{1}
\end{array}\right]
$$

Then a computation gives that

$$
\begin{aligned}
\operatorname{det} L & =\left(\lambda_{2} \bar{\lambda}_{1}-\lambda_{3} \bar{\lambda}_{2}\right)-\left(\lambda_{1} \bar{\lambda}_{1}-\lambda_{3} \bar{\lambda}_{3}\right)+\left(\lambda_{1} \bar{\lambda}_{2}-\lambda_{2} \bar{\lambda}_{3}\right) \\
& =\left(\lambda^{-2}-\lambda^{2}\right)+2\left(\lambda-\lambda^{-1}\right) \cos 3 \varphi .
\end{aligned}
$$

The equality det $L=0$ is equivalent to $\cos 3 \varphi=\left(\lambda+\lambda^{-1}\right) / 2$, which is impossible since $\lambda+\lambda^{-1}>2$.

It follows from Corollary 2.1 that every coefficient of the system lies in $\mathbb{Q}(\Gamma, \lambda)$. Solving this system by Cramer's rule, we conclude that every $b_{i i}$ lies in $\mathbb{Q}(\Gamma, \lambda)$. 
Lemma 2.3. Let $\Gamma$ be a subgroup of $\mathrm{SU}(2,1)$ containing a loxodromic element $A=\operatorname{diag}\left(\lambda_{1}, \lambda_{2}, \lambda_{3}\right)$, where $\lambda_{1}=\lambda e^{i \varphi}, \lambda_{2}=e^{-i 2 \varphi}, \lambda_{3}=\lambda^{-1} e^{i \varphi}$. Then for any element $B=\left(b_{i j}\right) \in \Gamma$, the products $b_{12} b_{21}, b_{13} b_{31}, b_{23} b_{32}$ are in $\mathbb{Q}(\Gamma, \lambda)$.

Proof. Let $C=B A B$. First, we compute the diagonal elements $c_{i i}$ of the matrix $C$. They are

$$
\begin{aligned}
& c_{11}=\lambda_{1} b_{11}^{2}+\lambda_{2} b_{12} b_{21}+\lambda_{3} b_{13} b_{31}, \\
& c_{22}=\lambda_{1} b_{12} b_{21}+\lambda_{2} b_{22}^{2}+\lambda_{3} b_{23} b_{32}, \\
& c_{33}=\lambda_{1} b_{13} b_{31}+\lambda_{2} b_{23} b_{32}+\lambda_{3} b_{33}^{2} .
\end{aligned}
$$

Then by applying Lemma 2.2, we have that $c_{i i}$ is in $\mathbb{Q}(\Gamma, \lambda)$. Also, by the same reason, $b_{i i}$ is in $\mathbb{Q}(\Gamma, \lambda)$. Therefore, we can re-write these equalities in the following form

$$
\begin{aligned}
& \lambda_{2} b_{12} b_{21}+\lambda_{3} b_{13} b_{31}=t_{1}, \\
& \lambda_{1} b_{12} b_{21}+\lambda_{3} b_{23} b_{32}=t_{2}, \\
& \lambda_{1} b_{13} b_{31}+\lambda_{2} b_{23} b_{32}=t_{3},
\end{aligned}
$$

where $t_{1}, t_{2}, t_{3}$ are some elements of $\mathbb{Q}(\Gamma, \lambda)$.

We consider these equalities as a system of linear equations in three unknowns $x_{1}=b_{12} b_{21}, x_{2}=b_{13} b_{31}, x_{3}=b_{23} b_{32}$. The matrix $L$ of this system is

$$
L=\left[\begin{array}{ccc}
\lambda_{2} & \lambda_{3} & 0 \\
\lambda_{1} & 0 & \lambda_{3} \\
0 & \lambda_{1} & \lambda_{2}
\end{array}\right] .
$$

A short computation shows that $\operatorname{det} L=-2 \lambda_{1} \lambda_{2} \lambda_{3}=-2 \operatorname{det} A \neq 0$. Solving this system by Cramer's rule, we conclude that every $x_{i}$ lies in $\mathbb{Q}(\Gamma, \lambda)$.

Lemma 2.4. Let $\Gamma$ be a subgroup of $\mathrm{SU}(2,1)$ containing a loxodromic element $A=\operatorname{diag}\left(\lambda_{1}, \lambda_{2}, \lambda_{3}\right)$, where $\lambda_{1}=\lambda e^{i \varphi}, \lambda_{2}=e^{-i 2 \varphi}, \lambda_{3}=\lambda^{-1} e^{i \varphi}$. Then for any element $B=\left(b_{i j}\right) \in \Gamma$, the products $b_{12} \bar{b}_{32}, b_{13} \bar{b}_{31}, b_{23} \bar{b}_{21}$ are in $\mathbb{Q}(\Gamma, \lambda)$.

Proof. Let $C=B A B^{-1}$. Let us compute the diagonal elements $c_{i i}$ of the matrix $C$. They are

$$
\begin{aligned}
& c_{11}=\lambda_{1} b_{11} \bar{b}_{33}+\lambda_{2} b_{12} \bar{b}_{32}+\lambda_{3} b_{13} \bar{b}_{31}, \\
& c_{22}=\lambda_{1} b_{21} \bar{b}_{23}+\lambda_{2} b_{22} \bar{b}_{22}+\lambda_{3} b_{23} \bar{b}_{21}, \\
& c_{33}=\lambda_{1} b_{31} \bar{b}_{13}+\lambda_{2} b_{32} \bar{b}_{12}+\lambda_{3} b_{33} \bar{b}_{11} .
\end{aligned}
$$

By applying Lemma 2.2, we get that $c_{i i}$ lies in $\mathbb{Q}(\Gamma, \lambda)$. Since $\lambda$ is real, we have that the field $\mathbb{Q}(\Gamma, \lambda)$ is invariant under complex conjugation. Hence, $b_{11} \bar{b}_{33}, b_{22} \bar{b}_{22}$, $b_{33} \bar{b}_{11}$ are all in $\mathbb{Q}(\Gamma, \lambda)$. Therefore, we can re-write these equalities in the following form

$$
\lambda_{2} b_{12} \bar{b}_{32}+\lambda_{3} b_{13} \bar{b}_{31}=t_{1},
$$




$$
\begin{aligned}
& \lambda_{1} b_{21} \bar{b}_{23}+\lambda_{3} b_{23} \bar{b}_{21}=t_{2}, \\
& \lambda_{1} b_{31} \bar{b}_{13}+\lambda_{2} b_{32} \bar{b}_{12}=t_{3},
\end{aligned}
$$

where $t_{1}, t_{2}, t_{3}$ are some elements of $\mathbb{Q}(\Gamma, \lambda)$.

Now let $D=B^{-1} A B$. Again we compute the diagonal elements $d_{i i}$ of this matrix. They are

$$
\begin{aligned}
& d_{11}=\lambda_{1} b_{11} \bar{b}_{33}+\lambda_{2} b_{21} \bar{b}_{23}+\lambda_{3} b_{31} \bar{b}_{13}, \\
& d_{22}=\lambda_{1} b_{12} \bar{b}_{32}+\lambda_{2} b_{22} \bar{b}_{22}+\lambda_{3} b_{32} \bar{b}_{12}, \\
& d_{33}=\lambda_{1} b_{13} \bar{b}_{31}+\lambda_{2} b_{23} \bar{b}_{21}+\lambda_{3} b_{33} \bar{b}_{11} .
\end{aligned}
$$

By applying the above arguments, we re-write these equalities in the following form

$$
\begin{aligned}
& \lambda_{2} b_{21} \bar{b}_{23}+\lambda_{3} b_{31} \bar{b}_{13}=s_{1}, \\
& \lambda_{1} b_{12} \bar{b}_{32}+\lambda_{3} b_{31} \bar{b}_{12}=s_{2}, \\
& \lambda_{1} b_{13} \bar{b}_{32}+\lambda_{2} b_{23} \bar{b}_{21}=s_{3},
\end{aligned}
$$

where $s_{1}, s_{2}, s_{3}$ are some elements of $\mathbb{Q}(\Gamma, \lambda)$.

We consider the first and the conjugate third equality defined by $C$ and the conjugate first equality defined by $D$. So, we have the following equalities:

$$
\begin{aligned}
& \lambda_{2} b_{12} \bar{b}_{32}+\lambda_{3} b_{13} \bar{b}_{31}=t_{1}, \\
& \bar{\lambda}_{1} b_{13} \bar{b}_{31}+\bar{\lambda}_{2} b_{12} \bar{b}_{32}=\bar{t}_{3}, \\
& \bar{\lambda}_{2} b_{23} \bar{b}_{21}+\bar{\lambda}_{3} b_{13} \bar{b}_{31}=\bar{s}_{1} .
\end{aligned}
$$

These equalities define a system of linear equations in three unknowns $x_{1}=b_{12} \bar{b}_{32}$, $x_{2}=b_{13} \bar{b}_{31}, x_{3}=b_{23} \bar{b}_{21}$. The matrix $L$ of this system is

$$
L=\left[\begin{array}{ccc}
\lambda_{2} & \lambda_{3} & 0 \\
\bar{\lambda}_{2} & \bar{\lambda}_{1} & 0 \\
0 & \bar{\lambda}_{3} & \bar{\lambda}_{2}
\end{array}\right]
$$

The determinant $\operatorname{det} L=\lambda_{2}\left(\bar{\lambda}_{1} \bar{\lambda}_{2}\right)-\lambda_{3} \bar{\lambda}_{2}^{2}=e^{2 i \varphi}\left(e^{-3 i \varphi} \lambda-e^{3 i \varphi} \lambda^{-1}\right)$. It is seen that $\operatorname{det} L=0$ if and only if $\lambda^{2}=e^{6 i \varphi}$. This is impossible since $\lambda>0, \lambda \neq 1$. Solving this system by Cramer's rule, we conclude that every $x_{i}$ lies in $\mathbb{Q}(\Gamma, \lambda)$.

The following proposition is crucial in the proof of our main result.

Proposition 2.1. Let $\Gamma=\langle A, B\rangle$ be an irreducible subgroup of $\mathrm{SU}(2,1)$, where $A$ is a loxodromic element with eigenvalues $\lambda_{1}=\lambda e^{i \varphi}, \lambda_{2}=e^{-2 i \varphi}, \lambda_{3}=\lambda^{-1} e^{i \varphi}$. Then $\Gamma$ is conjugate in $\mathrm{SU}(2,1)$ to a subgroup of $\mathrm{SU}(2,1, \mathbb{Q}(\Gamma, \lambda))$. 
Proof. We will show that one needs at most two conjugations to get the result we need.

First, by applying a suitable conjugation in $\mathrm{SU}(2,1)$, we may assume that

$$
A=\left[\begin{array}{ccc}
\lambda_{1} & 0 & 0 \\
0 & \lambda_{2} & 0 \\
0 & 0 & \lambda_{3}
\end{array}\right], \quad B=\left[\begin{array}{lll}
b_{11} & b_{12} & b_{13} \\
b_{21} & b_{22} & b_{23} \\
b_{31} & b_{32} & b_{33}
\end{array}\right] .
$$

By $v_{1}=\left(b_{11}, b_{12}, b_{13}\right)^{\top}, v_{2}=\left(b_{21}, b_{22}, b_{23}\right) \top$, and $v_{3}=\left(b_{31}, b_{32}, b_{33}\right)^{\top}$ we denote the vectors defined by the rows of the matrix $B$.

Let $X \in \mathrm{SU}(2,1)$ be a loxodromic element (elliptic if $r=1$ ) such that

$$
X=\left[\begin{array}{ccc}
r e^{i \alpha} & 0 & 0 \\
0 & e^{-2 i \alpha} & 0 \\
0 & 0 & r^{-1} e^{i \alpha}
\end{array}\right] .
$$

Then $X A X^{-1}=A$ and $X B X^{-1}$ is

$$
X B X^{-1}=\left[\begin{array}{ccc}
b_{11} & r e^{3 i \alpha} b_{12} & r^{2} b_{13} \\
r^{-1} e^{-3 i \alpha} b_{21} & b_{22} & r e^{-3 i \alpha} b_{23} \\
r^{-2} b_{31} & r^{-1} e^{3 i \alpha} b_{32} & b_{33}
\end{array}\right] .
$$

If the entries $b_{12}, b_{32}$ of $B$ are all equal to 0 , then the group $\Gamma$ is not irreducible since in this case $A$ and $B$ have a common invariant complex line spanned by the vector $(0,1,0)^{\top}$. In this case, $\Gamma$ is a $\mathbb{C}$-group. Therefore, at least one of the numbers $b_{12}, b_{32}$ is not equal to 0 . Let us suppose that $b_{12} \neq 0$. In this case, by normalizing the elements $A$ and $B$ using $X$, we may assume without loss of generality that $b_{12}=1$.

From the above results we know that $b_{i i}, b_{12} b_{21}, b_{13} b_{31}, b_{23} b_{32}, b_{12} \bar{b}_{32}, b_{13} \bar{b}_{31}$, $b_{23} \bar{b}_{21}$ are all in $\mathbb{Q}(\Gamma, \lambda)$.

Since $b_{12}=1$, we get that $b_{21}$ and $b_{32}$ are in $\mathbb{Q}(\Gamma, \lambda)$. Let us first consider the case $b_{21}=0$. Then we have that $b_{31} \neq 0$. Indeed, if $b_{31}=0$, then $A$ and $B$ have a common invariant complex line spanned by the vector $(1,0,0)^{\top}$. This is impossible because $\Gamma$ is irreducible. Let us assume now that $b_{32}=0$. Since the vectors $v_{2}$ and $v_{3}$ are orthogonal, we get that $\left\langle v_{2}, v_{3}\right\rangle=b_{23} \bar{b}_{31}=0$. So, in this case, $b_{23}=0$. This implies that $b_{22} \neq 0$. Hence, $\left\langle v_{1}, v_{2}\right\rangle=\bar{b}_{22} \neq 0$, a contradiction. Therefore, we have that $b_{32} \neq 0$. It follows that $b_{23}$ lies in $\mathbb{Q}(\Gamma, \lambda)$. Since the vectors $v_{1}$ and $v_{2}$ are orthogonal, we have that $b_{23} \neq 0$. Then, by considering the equality $\left\langle v_{2}, v_{3}\right\rangle=b_{22} \bar{b}_{32}+b_{23} \bar{b}_{31}=0$, we conclude that $b_{31}$ lies in $\mathbb{Q}(\Gamma, \lambda)$. Since $b_{31} \neq 0$, we get that $b_{13}$ lies in $\mathbb{Q}(\Gamma, \lambda)$. This shows that all the entries of the matrix $B$ are in $\mathbb{Q}(\Gamma, \lambda)$. Therefore, we proved the proposition in this case.

Now let us assume that $b_{21} \neq 0$. It follows immediately that $b_{23}$ lies in $\mathbb{Q}(\Gamma, \lambda)$.

We write $\left\langle v_{1}, v_{2}\right\rangle=b_{11} \bar{b}_{23}+\bar{b}_{22}+b_{13} \bar{b}_{21}=0$. Since $b_{21} \neq 0$, this implies that $b_{13}$ is in $\mathbb{Q}(\Gamma, \lambda)$. If $b_{13} \neq 0$, we get that $b_{31}$ is in $\mathbb{Q}(\Gamma, \lambda)$. Suppose that $b_{13}=0$. In this case, $b_{23} \neq 0$, since otherwise $A$ and $B$ have a common invariant complex line spanned by the vector $(0,0,1)^{\top}$. This is impossible because $\Gamma$ is irreducible. 
We write $\left\langle v_{2}, v_{3}\right\rangle=b_{21} \bar{b}_{33}+b_{22} \bar{b}_{32}+b_{23} \bar{b}_{31}=0$. Since $b_{23} \neq 0$, we get that $b_{31}$ lies in $\mathbb{Q}(\Gamma, \lambda)$.

Summarizing everything, we get that in the case $b_{12} \neq 0$ after conjugation all the entries of the matrix $B$ lie in $\mathbb{Q}(\Gamma, \lambda)$. The case $b_{12}=0, b_{32} \neq 0$ is similar to the previous one.

Next we prove that any irreducible subgroup of $\mathrm{SU}(2,1)$ contains a loxodromic element. First, we will prove the following lemma.

Lemma 2.5. Let $\Gamma$ be a non-elementary subgroup of $\mathrm{SU}(2,1)$. If $\Gamma$ contains a parabolic element, then $\Gamma$ contains a loxodromic element.

Proof. Let $B \in \Gamma$ be parabolic. Then $B$ has a unique invariant isotropic complex line in $V$. By normalizing $\Gamma$ in $\mathrm{SU}(2,1)$, we may assume without loss of generality that this line is spanned by the vector $(1,0,0)^{\top}$. This implies that $B$ has the following form:

$$
B=\left[\begin{array}{ccc}
b_{11} & b_{12} & b_{13} \\
0 & b_{22} & b_{23} \\
0 & b_{32} & b_{33}
\end{array}\right] .
$$

As before, we denote by $v_{1}=\left(b_{11}, b_{12}, b_{13}\right)^{\top}, v_{2}=\left(b_{21}, b_{22}, b_{23}\right)^{\top}, v_{3}=$ $\left(b_{31}, b_{32}, b_{33}\right)^{\top}$ the vectors defined by the rows of the matrix $B$.

We consider two cases: (1) $b_{12}=0$, (2) $b_{12} \neq 0$.

First, we consider the case $b_{12}=0$. We have that $\left\langle v_{2}, v_{2}\right\rangle=\left|b_{22}\right|^{2}=1$. From this and the equality $\left\langle v_{2}, v_{3}\right\rangle=0$, we get that $b_{32}=0$. Then the equality $\left\langle v_{1}, v_{2}\right\rangle=b_{11} \bar{b}_{23}=0$ implies that $b_{23}=0$. From the equality $\left\langle v_{1}, v_{3}\right\rangle=1$, we get that $b_{11} \bar{b}_{33}=1$. Since $B$ is parabolic, we have that $b_{13} \neq 0$ and that $\left|b_{11}\right|=1$. Hence $\left|b_{33}\right|=1$. We write $b_{11}=e^{i \varphi}$ and $b_{13}=r e^{i \theta}, r>0$. Then the equality $\left\langle v_{1}, v_{1}\right\rangle=0$ implies that $b_{13}=r i e^{i \varphi}$. Therefore, when $b_{12}=0$, the element $B$ after a suitable normalization of $\Gamma$ has the following form:

$$
B=\left[\begin{array}{ccc}
e^{i \varphi} & 0 & r i e^{i \varphi} \\
0 & e^{-2 i \varphi} & 0 \\
0 & 0 & e^{i \varphi}
\end{array}\right]
$$

We remark that if $\varphi=0$, then $B$ is unipotent and $B$ is ellipto-parabolic otherwise.

Next, we consider the case $b_{12} \neq 0$. Using the same arguments as in the first case, we get that $\left|b_{22}\right|=1$ and $b_{32}=0$. Since $b_{12} \neq 0$, by normalizing $\Gamma$ using the element $X$ defined in the proof of Proposition 2.1, we may assume without loss of generality that $b_{12}=1$. The equality $\left\langle v_{1}, v_{3}\right\rangle=1$ implies that $b_{11} \bar{b}_{33}=1$. Note that in this case $B$ is always unipotent. Hence $b_{11}=1$. This implies that $b_{22}=b_{33}=1$. Then it follows from the equality $\left\langle v_{1}, v_{2}\right\rangle=0$ that $b_{23}=-1$. Now let us consider the equality $\left\langle v_{1}, v_{1}\right\rangle=\bar{b}_{13}+b_{13}+1=0$. It easy to see that this equality is true if and only if $b_{13}=-1 / 2+s i$, where $s$ is real. Summarizing everything, we get 
that in the case $b_{12} \neq 0$ after a suitable normalization of $\Gamma$ the element $B$ has the following form:

$$
B=\left[\begin{array}{ccc}
1 & 1 & \tau \\
0 & 1 & -1 \\
0 & 0 & 1
\end{array}\right]
$$

where $\tau=-1 / 2+s i, s \in \mathbb{R}$.

An easy induction shows that for any $n \in \mathbb{N}$ in the first case

$$
B^{n}=\left[\begin{array}{ccc}
e^{n i \varphi} & 0 & e^{n i \varphi} i n r \\
0 & e^{-2 n i \varphi} & 0 \\
0 & 0 & e^{n i \varphi}
\end{array}\right]
$$

and

$$
B^{n}=\left[\begin{array}{ccc}
1 & n & n \tau+f(n) \\
0 & 1 & -n \\
0 & 0 & 1
\end{array}\right]
$$

in the second case, where the function $f: \mathbb{N} \rightarrow \mathbb{Z}$ is defined by the following conditions: $f(1)=0$ and $f(n+1)=f(n)-n$. It is easy to show that $f(n)=$ $(1-n) n / 2$.

Since $\Gamma$ is non-elementary it contains an element $C$ which does not leave invariant the complex line spanned by the vector $(1,0,0)^{\top}$. Let $C=\left(c_{i j}\right)$. Then we have that $c_{31} \neq 0$. Easy computation shows that

$$
\operatorname{tr}\left(B^{n} C\right)=c_{11} e^{n i \varphi}+c_{31} e^{n i \varphi} i n r+c_{22} e^{-2 n i \varphi}+c_{33} e^{n i \varphi}
$$

in the first case, and

$$
\operatorname{tr}\left(B^{n} C\right)=c_{11}+c_{21} n+c_{31}(n \tau+f(n))+c_{22}-c_{32} n+c_{33}
$$

in the second one.

Hence, in the first case

$$
\begin{aligned}
\left|\operatorname{tr}\left(B^{n} C\right)\right| & =\left|c_{31} e^{n i \varphi} i n r-\left(-c_{11} e^{n i \varphi}-c_{22} e^{-2 n i \varphi}-c_{33} e^{n i \varphi}\right)\right| \\
& \geq\left|c_{31} e^{n i \varphi} i n r\right|-\left|\left(-c_{11} e^{n i \varphi}-c_{22} e^{-2 n i \varphi}-c_{33} e^{n i \varphi}\right)\right| \\
& =\left|c_{31}\right| n r-\left|\left(-c_{11} e^{n i \varphi}-c_{22} e^{-2 n i \varphi}-c_{33} e^{n i \varphi}\right)\right| .
\end{aligned}
$$

Since $c_{31} \neq 0$, and $r>0$, this inequality implies that $\left|\operatorname{tr}\left(B^{n} C\right)\right|$ tends to infinity when $n$ tends to infinity.

In the second case, we have that

$$
\begin{aligned}
\left|\operatorname{tr}\left(B^{n} C\right)\right| & =\left|c_{11}+c_{21} n+c_{31}(n \tau+f(n))+c_{22}-c_{32} n+c_{33}\right| \\
& \geq\left|c_{31} f(n)\right|-\left|-c_{31} n \tau-c_{11}-c_{21} n-c_{22}+c_{32} n-c_{33}\right| \\
& =\left|c_{31}\right||f(n)|-\left|-c_{31} n \tau-c_{11}-c_{21} n-c_{22}+c_{32} n-c_{33}\right| .
\end{aligned}
$$


Since $c_{31} \neq 0$ and $f(n)$ is quadratic, this inequality implies that $\left|\operatorname{tr}\left(B^{n} C\right)\right|$ tends to infinity when $n$ tends to infinity.

Thus, in both cases, we have that there exists $n_{0}$ such that $\left|\operatorname{tr}\left(B^{n} C\right)\right|>3$ for all $n>n_{0}$. Then using Goldman's classification of the elements in $\mathrm{SU}(2,1)[16]$, we get that the elements $B^{n} C$ are loxodromic for all $n>n_{0}$. This proves the lemma.

We are very grateful to John Parker for his help in the proof of this lemma.

In what follows, we will need the following fundamental result due to Chen and Greenberg [4]:

Proposition 2.2. Let $\Gamma$ be a subgroup of $\mathrm{SU}(2,1)$. Consider the natural action of $\Gamma$ on $\boldsymbol{H}_{\mathbb{C}}^{2} \cup \partial \boldsymbol{H}_{\mathbb{C}}^{2}$. If there is no point in $\boldsymbol{H}_{\mathbb{C}}^{2} \cup \partial \boldsymbol{H}_{\mathbb{C}}^{2}$ or proper totally geodesic submanifold in $\boldsymbol{H}_{\mathbb{C}}^{2}$ which is invariant under $\Gamma$, then $\Gamma$ is either discrete or dense in $\mathrm{SU}(2,1)$.

By applying this result and Lemma 2.5, we prove the following proposition.

Proposition 2.3. Let $\Gamma$ be an irreducible subgroup of $\mathrm{SU}(2,1)$. Then $\Gamma$ contains a loxodromic element.

Proof. Let us consider the natural action of $\Gamma$ on the projective space $\mathbb{P}(V)$ (or equally the action of its projectivization). We know that the only proper totally geodesic submanifolds of $\boldsymbol{H}_{\mathbb{C}}^{2}$ are either geodesics, or complex geodesics, or totally real geodesic 2-planes [4], [16]. Note that if there is a geodesic in $\boldsymbol{H}_{\mathbb{C}}^{2}$ which is invariant under $\Gamma$, then there is a complex geodesic $c$ in $\boldsymbol{H}_{\mathbb{C}}^{2}$ which is also invariant under $\Gamma$ (this complex geodesic $c$ is spanned by the geodesic in question). Therefore, the polar point to $c$ is invariant under $\Gamma$ for its action on the projective space $\mathbb{P}(V)$. Hence, if $\Gamma$ is irreducible, then either $\Gamma$ has no invariant proper totally geodesic submanifolds or $\Gamma$ is an $\mathbb{R}$-subgroup of $\mathrm{SU}(2,1)$.

First, we consider the case when $\Gamma$ has no invariant totally real geodesic 2-planes. By applying Proposition 2.2, we get that $\Gamma$ is either discrete or dense in $\mathrm{SU}(2,1)$. If $\Gamma$ is dense in $\mathrm{SU}(2,1)$, it contains a loxodromic element since the set of loxodromic elements is open in $\operatorname{SU}(2,1)[16]$, and we are done. Now let us assume that $\Gamma$ is a discrete subgroup of $\mathrm{SU}(2,1)$. Suppose that $\Gamma$ has no loxodromic elements. If $\Gamma$ contains only elliptic elements, then it is easy to see that $\Gamma$ is finite, and then, using the arguments similar to those in [25], we conclude that there is a point in $\boldsymbol{H}_{\mathbb{C}}^{2}$ which is invariant under $\Gamma$, a contradiction. Therefore, $\Gamma$ contains a parabolic element $\gamma$. Since $\Gamma$ is irreducible, it contains an element not fixing the unique fixed point of $\gamma$. By applying Lemma 2.5 , we get that $\Gamma$ contains a loxodromic element, a contradiction.

Now, let $\Gamma$ be an $\mathbb{R}$-subgroup. Then $\Gamma$ is conjugate in $\operatorname{SU}(2,1)$ to a subgroup of $\mathrm{SO}(2,1)$. Since $\Gamma$ irreducible, the result follows from the plane real hyperbolic geometry [2], [25]. 
Corollary 2.2. Let $G$ be an irreducible subgroup of $\mathrm{PU}(2,1)$. Then $G$ is nonelementary.

Proof. Assume that $G$ is elementary. Let $p \in \boldsymbol{H}_{\mathbb{C}}^{2} \cup \partial \boldsymbol{H}_{\mathbb{C}}^{2}$ be a point such that the orbit $G(p)$ of $p$ is finite. If $p \in \boldsymbol{H}_{\mathbb{C}}^{2}$, then it follows from the proof of Proposition 2.3 that there is a point in $\boldsymbol{H}_{\mathbb{C}}^{2}$ which is invariant under $\Gamma$, a contradiction. Next, let us suppose that $p \in \partial \boldsymbol{H}_{\mathbb{C}}^{2}$. By applying Proposition 2.3, we have that $G$ contains a loxodromic element. Since $G(p)$ is finite, this implies that $G(p)$ consists of two distinct points. Let $G(p)=\left\{p_{1}, p_{2}\right\}$ and $c$ be the unique complex geodesic spanned by $\left\{p_{1}, p_{2}\right\}$. Then $c$ is invariant under $G$, and, therefore, $G$ leaves invariant its polar point, a contradiction.

Thus, we proved that any irreducible subgroup of $\mathrm{SU}(2,1)$ contains a loxodromic element. With this, we now prove our main result: if $\Gamma$ is an irreducible subgroup of $\mathrm{SU}(2,1)$, then $\Gamma$ is conjugate in $\mathrm{SU}(2,1)$ to a subgroup of $\mathrm{SU}(2,1, \mathbb{Q}(\Gamma, \lambda))$, where $\lambda$ is the absolute value of an eigenvalue of any loxodromic element of $\Gamma$ with $|\lambda| \neq 1$. In particular, this implies that $\Gamma$ can be defined over the field $\mathbb{Q}(\Gamma, \lambda)$.

Theorem 2.1. Let $\Gamma$ be an irreducible subgroup of $\mathrm{SU}(2,1)$. Let $A \in \Gamma$ be any loxodromic element with eigenvalues $\lambda_{1}=\lambda e^{i \varphi}, \lambda_{2}=e^{-2 i \varphi}, \lambda_{3}=\lambda^{-1} e^{i \varphi}$. Then $\Gamma$ is conjugate in $\mathrm{SU}(2,1)$ to a subgroup of $\mathrm{SU}(2,1, \mathbb{Q}(\Gamma, \lambda))$.

Proof. First, we show that $\Gamma$ contains two loxodromic elements $A_{1}$ and $A_{2}$ having the same eigenvalues as $A$ (in fact, we show that $A_{1}$ and $A_{2}$ are conjugate to $A$ in $\Gamma)$ such that the subgroup $\Gamma_{0}=\left\langle A_{1}, A_{2}\right\rangle$ generated by $A_{1}$ and $A_{2}$ is irreducible. In what follows, we consider the natural action of $\Gamma$ on the projective space $\mathbb{P}(V)$. We remark that $A$ has three fixed points $p_{1}, p_{2}, p_{3}$ for its action on the projective space: $p_{1}, p_{2}$ are isotropic and $p_{3}$ is positive, $p_{3}$ is the polar point to the complex projective line $\alpha$ spanned by $p_{1}$ and $p_{2}$. If all the elements of $\Gamma$ fix the point $p_{3}$, then $\Gamma$ is reducible. So, there exists an element $B$ of $\Gamma$ which does not fix $p_{3}$. Let $C=B A B^{-1}$ and $q_{3}=B\left(p_{3}\right)$. Then $C\left(q_{3}\right)=q_{3}$. Note that $C$ is loxodromic, hence $C$ does not fix $p_{3}$. Let $q_{1}$ and $q_{2}$ be the isotopic fixed point of $C$. If the sets $\left\{p_{1}, p_{2}\right\}$ and $\left\{q_{1}, q_{2}\right\}$ are disjoint, taking $A_{1}=A$ and $A_{2}=C$ we are done, since in this case the elements $A$ and $C$ have no common fixed points for their action on $\mathbb{P}(V)$. So, let us consider the case when these sets have non-empty intersection, that is, when the elements $A$ and $C$ have a common isotropic fixed point. If $\left\{p_{1}, p_{2}\right\}=\left\{q_{1}, q_{2}\right\}$, then the complex projective line $\alpha$ is invariant under $C$, and, therefore, $C\left(p_{3}\right)=p_{3}$, a contradiction. This implies that $A$ and $C$ may have only one common isotropic fixed point. One may assume without loss of generality that $p_{1}=q_{1}$ is a unique common isotropic fixed point of $A$ and $C$. Then since $\Gamma$ is irreducible, there exists an element $D \in \Gamma$ which does not fix the point $p_{1}$. Let $E=D A D^{-1}$. We have that $E$ is loxodromic and $E\left(p_{1}\right) \neq p_{1}$. If the invariant complex geodesic $\beta$ of $E$ is not equal to $\alpha$, then taking $A_{1}=A$ and $A_{2}=E$, we are done. If $\alpha=\beta$, we take $A_{1}=C$ and $A_{2}=E$. 
Now, by applying Proposition 2.1, we get that there exists $f \in \mathrm{SU}(2,1)$ such that $\Gamma_{0}^{*}=f \Gamma_{0} f^{-1}$ is a subgroup of $\operatorname{SU}\left(2,1, \mathbb{Q}\left(\Gamma_{0}, \lambda\right)\right)$. Let $\Gamma^{*}=f \Gamma f^{-1}$. Then $\Gamma_{0}^{*}$ is a subgroup of $\Gamma^{*}$. In order to show that $\Gamma^{*}$ is a subgroup of $\operatorname{SU}(2,1, \mathbb{Q}(\Gamma, \lambda))$, we apply Burnside's density theorem, stated as Theorem 16" in I. Kaplansky's book [20], and the trick which one can find in the proof of Theorem B in [20]. According to Kaplansky this trick is due to C. Procesi.

Since $\Gamma_{0}^{*}$ is irreducible, by applying Burnside's density theorem, we get that $\Gamma_{0}^{*}$ contains a basis of $\mathrm{M}(3, \mathbb{C})$ over $\mathbb{C}$. Let $S=\left\{S_{1}, S_{2}, \ldots, S_{9}\right\}$ be such a basis. Then it follows that for any element $\gamma \in \Gamma^{*}$ there exist complex numbers $c_{1}, c_{2}, \ldots, c_{9}$ such that

$$
\gamma=c_{1} S_{1}+c_{2} S_{2}+\ldots+c_{9} S_{9} .
$$

Let $\operatorname{Tr}(A, B)=(A, B)$ denote the trace form on $\mathrm{M}(3, \mathbb{C})$ so that

$$
(A, B)=\operatorname{Tr}(A, B)=\operatorname{tr}(A B) .
$$

It is well known that $\operatorname{Tr}$ is a non-degenerate symmetric bilinear form.

It follows from the equality

$$
\gamma=c_{1} S_{1}+c_{2} S_{2}+\ldots+c_{9} S_{9}
$$

that

$$
\left(\gamma, S_{i}\right)=c_{1}\left(S_{1}, S_{i}\right)+c_{2}\left(S_{2}, S_{i}\right)+\ldots+c_{9}\left(S_{9}, S_{i}\right)
$$

for all $i=1,2, \ldots, 9$.

We consider these equalities as a system of linear equations in unknowns $c_{1}, c_{2}, \ldots, c_{9}$. We have that for all $i, j=1,2, \ldots, 9$, the product $\left(S_{i}, S_{j}\right)$ is in $\mathbb{Q}\left(\Gamma_{0}, \lambda\right)$, and the product $\left(\gamma, S_{i}\right)$ is in $\mathbb{Q}(\Gamma, \lambda)$. Therefore, this implies that every coefficient of the system lies in $\mathbb{Q}(\Gamma, \lambda)$. Since the form $\operatorname{Tr}$ is non-degenerate, this system is non-singular. Solving this system by Cramer's rule, we conclude that every $c_{i}$ lies in $\mathbb{Q}(\Gamma, \lambda)$. This implies that every $\gamma \in \Gamma^{*}$ lies in $\mathrm{SU}(2,1, \mathbb{Q}(\Gamma, \lambda))$. From this, we conclude that $\Gamma^{*}$ is a subgroup of $\operatorname{SU}(2,1, \mathbb{Q}(\Gamma, \lambda))$.

As a corollary of this theorem, we get the following.

Theorem 2.2. Let $\Gamma$ be an irreducible subgroup of $\mathrm{SU}(2,1)$ such that $\mathbb{Q}(\Gamma)$ is a subset of $\mathbb{R}$, then $\Gamma$ is conjugate in $\mathrm{SU}(2,1)$ to a subgroup of $\mathrm{SO}(2,1)$.

Proof. Since $\Gamma$ is irreducible, it follows from Proposition 2.3 that $\Gamma$ contains a loxodromic element $A$. Let $\lambda_{1}=\lambda e^{i \varphi}, \lambda_{2}=e^{-2 i \varphi}, \lambda_{3}=\lambda^{-1} e^{i \varphi}$ be its eigenvalues. It is easy to see that in this case all the eigenvalues of $A$ are real: $A$ is either hyperbolic or loxodromic whose elliptic part is of order 2. Since the field $\mathbb{Q}(\Gamma)$ is real, the field $\mathbb{Q}(\Gamma, \lambda)$ is also real. So, the result follows from Theorem 2.1. 
We would like to stress that in Theorem 2.1 and Theorem 2.2 we do not assume that the group $\Gamma$ is discrete.

Next we show that the conclusions of Theorem 2.1 and Theorem 2.2 are not true if $\Gamma$ is reducible. Indeed, let us consider the embedding of $\operatorname{SL}(2, \mathbb{R})$ in $\mathrm{SU}(2,1)$ given by

$$
\left[\begin{array}{ll}
a & b \\
c & d
\end{array}\right] \longrightarrow\left[\begin{array}{ccc}
a & 0 & -i b \\
0 & 1 & 0 \\
i c & 0 & d
\end{array}\right]
$$

This defines a faithful representation of $\operatorname{SL}(2, \mathbb{R})$ in $\operatorname{SU}(2,1)$. Let $\Gamma$ be the image of $\operatorname{SL}(2, \mathbb{R})$ under this embedding. Then $\Gamma$ is reducible because the complex line spanned by the vector $(0,1,0)^{\top}$ is invariant under $\Gamma$. In fact, $\Gamma$ is a $\mathbb{C}$-subgroup of $\mathrm{SU}(2,1)$. Also, we have that the trace field of $\Gamma$ is real. It is easy to see that $\Gamma$ cannot be conjugate in $\mathrm{SU}(2,1)$ to a subgroup of $\mathrm{SO}(2,1)$. Moreover, if we consider the image of $\operatorname{SL}(2, \mathbb{Z})$ under this embedding, we get an example of a non-elementary discrete subgroup of $\mathrm{SU}(2,1)$ whose trace field is $\mathbb{Q}$ but which is not conjugate in $\mathrm{SU}(2,1)$ to a subgroup of $\mathrm{SO}(2,1)$.

Now we consider the case of subgroups of $\mathrm{PU}(2,1)$. We would like to find conditions under which a subgroup $G$ of $\mathrm{PU}(2,1)$ leaves invariant a totally real geodesic 2-plane in $\boldsymbol{H}_{\mathbb{C}}^{2}$. It is natural to ask if it is possible or not to get an answer in terms of the traces of lifts of elements of $G$ to $\mathrm{SU}(2,1)$.

Let $\pi: \mathrm{SU}(2,1) \rightarrow \mathrm{PU}(2,1)$ be a natural projection. Let $G$ be a subgroup of PU $(2,1)$. Then the trace field of $G$, denoted $\mathbb{Q}(G)$, is the field $\mathbb{Q}(\Gamma)$, where $\Gamma=\pi^{-1}(G)$. We remark that this field is never real.

The following example is useful to understand the problem. Let $\Gamma$ be a subgroup of $\mathrm{SO}(2,1)$ such that the restriction $\pi: \Gamma \rightarrow \mathrm{PU}(2,1)$ is a monomorphism. Let $G=\pi(\Gamma)$. We have that $\Gamma$ is a lift of $G$. One could define an "invariant" trace field of $G$ as the trace field of $\Gamma$. And, in this case, this field is real! So, it would be natural to define an "invariant" trace field of a subgroup $G$ of $\mathrm{PU}(2,1)$ which has a lift to $\mathrm{SU}(2,1)$ as the trace field of its lift. Unfortunately, the problem when a subgroup $G$ of $\mathrm{PU}(2,1)$ has a lift to $\mathrm{SU}(2,1)$ is still open, and it seems very difficult. To our knowledge, the only results in this direction are in [17], in the case when $G$ is isomorphic to the fundamental group of a closed orientable surface. Some examples when $G$ has no lift, the reader can find in [1]. The lifting problem in the case of real hyperbolic geometry of dimension three was completely solved in [5].

In [26], [21], [28] the invariant trace field was defined for subgroups of PSL $(2, \mathbb{C})$, see also [23], for the case of $\mathrm{SU}(n, 1)$. We follow their ideas to define an invariant trace field for subgroups of $\mathrm{PU}(2,1)$.

Let $G$ be a subgroup of $\mathrm{PU}(2,1)$ and $\Gamma=\pi^{-1}(G)$. Then the invariant trace field of $G$, denoted by $k(G)$, is defined to be the field $\mathbb{Q}\left(\Gamma^{3}\right)$, where $\Gamma^{3}=\left\langle\gamma^{3}: \gamma \in \Gamma\right\rangle$. It follows from [23] that the invariant trace field is an invariant of the commensurability class. 
If $A \in \mathrm{SU}(2,1)$, then we have the following trace identity:

$$
\operatorname{tr}\left(A^{3}\right)=(\operatorname{tr}(A))^{3}-3 \operatorname{tr}(A) \operatorname{tr}\left(A^{-1}\right)+3,
$$

see, for instance, [24]. Since an element of PU(2, 1) has three lifts to $\operatorname{SU}(2,1)$ which differ by a cube root of unity, it follows from this formula that if $G$ is an $\mathbb{R}$-subgroup of $\mathrm{PU}(2,1)$, then the invariant trace field of $G$ is real.

By applying Theorem 2.2 , we get the following characterization of discrete $\mathbb{R}$ subgroups of $\mathrm{PU}(2,1)$.

Theorem 2.3. Let $G$ be an irreducible discrete subgroup of $\mathrm{PU}(2,1)$. Then $G$ is an $\mathbb{R}$-subgroup if and only if the invariant trace field $k(G)$ of $G$ is real.

Proof. Let $\Gamma=\pi^{-1}(G)$. Then it is clear that $\Gamma$ and $\Gamma^{3}$ are irreducible. Let $H=$ $\pi\left(\Gamma^{3}\right)$. It follows from Theorem 2.2 that $\Gamma^{3}$ is conjugate in $\operatorname{SU}(2,1)$ to a subgroup of $\mathrm{SO}(2,1)$. This implies that $H$ leaves invariant a totally real geodesic 2-plane $L$ in $\boldsymbol{H}_{\mathbb{C}}^{2}$. Since $\Gamma^{3}$ is a normal subgroup of $\Gamma$, it follows that $H$ is a normal subgroup of $G$. Since the group $G$ is irreducible, it follows from Corollary 2.2 that $G$ is nonelementary. Therefore, the normality implies that the limit set of $H$ is equal to the limit set of $G$, see, for instance, [4]. We remark that the limit set of $H$ is contained in the boundary of $L$. Moreover, using the fact that $G$ is non-elementary, we have that the set of fixed points of loxodromic elements of $G$ is dense in its limit set. This implies that $L$ is invariant with respect to $G$. Therefore, $G$ is an $\mathbb{R}$-subgroup of $\mathrm{PU}(2,1)$.

As an immediate corollary we get the following theorem.

Theorem 2.4. Let $G$ be a discrete non-elementary subgroup of $\mathrm{PU}(2,1)$ such that the invariant trace field $k(G)$ of $G$ is real. Then $G$ is either $\mathbb{R}$-Fuchsian or $\mathbb{C}$-Fuchsian.

Proof. If $G$ is irreducible, then it follows from Theorem 2.3 that $G$ is $\mathbb{R}$-Fuchsian. Now let us assume that $G$ is reducible. Let $p$ be a common fixed point of all elements of $G$ in their action on the projective space $\mathbb{P}(V)$. Since $G$ is non-elementary, we have that $p$ cannot be in $\boldsymbol{H}_{\mathbb{C}}^{2} \cup \partial \boldsymbol{H}_{\mathbb{C}}^{2}$. So, $p$ is positive. This implies that $G$ leaves invariant the complex geodesic whose polar point is $p$. Therefore, the group $G$ is $\mathbb{C}$-Fuchsian.

We remark that Theorem 2.4 can be considered as a complex hyperbolic analog of a classical result due to B.Maskit, see Theorem G.18 in [22] and Corollary 3.2.5 in [21].

We say that $g \in \mathrm{PU}(2,1)$ is a screw motion iff any lift of $g$ to $\mathrm{SU}(2,1)$ has non-real trace. For instance, purely hyperbolic and unipotent parabolic elements are not screw motions. Geometrically, $g \in \mathrm{PU}(2,1)$ is not screw motion iff $g$ has an invariant totally real geodesic 2-plane. 
Corollary 2.3. Let $G$ be an irreducible discrete subgroup of $\mathrm{PU}(2,1)$. Then $G$ is an $\mathbb{R}$-subgroup if and only if $G$ contains no screw motions.

Corollary 2.4. Let $G$ be an irreducible discrete subgroup of $\mathrm{PU}(2,1)$ whose limit set is not contained in an $\mathbb{R}$-circle. Then its invariant trace field is non-real extension of $\mathbb{Q}$.

Corollary 2.5. Let $G$ be a discrete subgroup of $\mathrm{PU}(2,1)$ of finite co-volume. Then its invariant trace field is non-real extension of $\mathbb{Q}$.

Proof. It is clear that $G$ is irreducible. Suppose that the invariant trace field $k(G)$ of $G$ is real. By Theorem 2.3, $G$ is an $\mathbb{R}$-subgroup of $\mathrm{PU}(2,1)$. Therefore, the limit set of $G$ is contained in an $\mathbb{R}$-circle. This implies that $G$ cannot have finite co-volume.

Acknowledgements. We are very grateful to John Parker for helpful conversations. We are also thankful to the referee for useful remarks and suggestions.

\section{References}

[1] S. Anan'in, C. Grossi, and N. Gusevskii, Complex hyperbolic structures on disc bundles over surfaces. Internat. Math. Res. Notices 2011 (2011), no. 19, 4295-4375. Zbl 1228.32024 MR 2838042

[2] A. Beardon, The geometry of discrete groups. Grad. Texts in Math. 91, Springer-Verlag, New York 1983. Zbl 0528.30001 MR 0698777

[3] J. Bernik, On groups and semigroups of matrices with spectra in a finitely generated field. Linear Multilinear Algebra 53 (2005), no. 4, 259-267. Zbl 1085.15020 MR 2160409

[4] S. S Chen and L. Greenberg, Hyperbolic spaces. In Contributions to analysis (a collection of papers dedicated to Lipman Bers), Academic Press, New York 1974, 49-87. Zbl 0295.53023 MR 0377765

[5] M. Culler, Lifting representations to covering groups. Adv. in Math. 59 (1986), no. 1, 64-70. Zbl 0582.57001 MR 0825087

[6] H. Cunha and N. Gusevskii, On the moduli space of quadruples of points in the boundary of complex hyperbolic space. Transform. Groups 15 (2010), no. 2, 261-283. Zbl 1205.53075 MR 2657443

[7] H. Cunha and N. Gusevskii, The moduli space of points in the boundary of complex hyperbolic space. J. Geom. Anal. 22 (2012), no. 1, 1-11. Zbl 1254.32039 MR 2868954

[8] H. Cunha, F. Dutenhefner, N. Gusevskii, and R. Thebadi, The moduli space of complex geodesics in the complex hyperbolic plane. J. Geom. Anal. 22 (2012), no. 2, 295-319. Zbl 1254.32038 MR 2891727

[9] F. Dutenhefner and N. Gusevskii, Complex hyperbolic Kleinian groups with limit set a wild knot. Topology 43 (2004), 677-696. Zbl 1051.30038 MR 2041637 
[10] D. Epstein, Complex hyperbolic geometry. In Analytical and geometric aspects of hyperbolic space (Coventry/Durham, 1984), London Math. Soc. Lecture Note Ser. 111, Cambridge University Press, Cambridge 1987, 93-111. Zbl 0611.51012 MR 0903851

[11] E. Falbel, F. Gabor, P. D. Lax, and J. R. Parker, Generators of a Picard modular group in two complex dimensions. Proc. Amer. Math. Soc. 139 (2011), no. 7, 2439-2447. Zbl 1222.32038 MR 2784810

[12] E. Falbel, F. Gabor, and J. R. Parker, The geometry of the Gauss-Picard modular group. Math. Ann. 349 (2011), no. 2, 459-508. Zbl 1213.14049 MR 2753829

[13] E. Falbel and J. R. Parker, The geometry of the Eisenstein-Picard modular group. Duke Math. J. 131 (2006), no. 2, 249-289. Zbl 1109.22007 MR 2219242

[14] X. Fu, L. Li, and X. Wang, A characterization of Fuchsian groups acting on complex hyperbolic spaces. Czechoslovak Math. J. 62 (137) (2012), no. 2, 517-525. Zbl 1265.30182 MR 2990191

[15] J. Genzmer, Trace fields of subgroups of SU(n, 1). Preprint, arXiv:1106.5986v1.

[16] W. M. Goldman, Complex hyperbolic geometry. Oxford Math. Monogr., Oxford Science Publications, The Clarendon Press, Oxford University Press, New York 1999. Zbl 0939.32024 MR 1695450

[17] W. M. Goldman, M. Kapovich, and B. Leeb, Complex hyperbolic manifolds homotopy equivalent to a Riemann surface. Comm. Anal. Geom. 9 (2001), no. 1, 61-95. Zbl 0982.32024 MR 1807952

[18] N. Gusevskii and J. R. Parker, Representations of free Fuchsian groups in complex hyperbolic space. Topology 39 (2000), no. 1, 33-60. Zbl 0977.32017 MR 1710991

[19] N. Gusevskii and J. R. Parker, Complex hyperbolic quasi-Fuchsian groups and Toledo's invariant. Geom. Dedicata 97 (2003), 151-185. Zbl 1042.57023 MR 2003696

[20] I. Kaplansky, Fields and rings. Second edition. Chicago Lectures in Mathematics, The University of Chicago Press, Chicago, Ill., London 1972. Zbl 1001.16500 MR 0349646

[21] C. Maclachlan and A. W. Reid, The arithmetic of hyperbolic 3-manifolds. Grad. Texts in Math. 219, Springer-Verlag, New York 2003. Zbl 1025.57001 MR 1937957

[22] B. Maskit, Kleinian groups. Grundlehren Math. Wiss. 287, Springer-Verlag, Berlin, 1988. Zbl 0627.30039 MR 0959135

[23] D. B. McReynolds, Arithmetic lattices in $\mathrm{SU}(n, 1)$. Book in preparation, available at http://www.its.caltech.edu/ dmcreyn/ComplexArithmeticI.pdf

[24] J. R. Parker, Traces in complex hyperbolic geometry. In Geometry, topology and dynamics of character varieties, Lect. Notes Ser. Inst. Math. Sci. Natl. Univ. Singapore 23, World Scientic Publishing, Hackensack, NJ, 2012, 191-245. Zbl 1269.51006 MR 2987619

[25] J. G. Ratcliffe, Foundations of hyperbolic manifolds. Grad. Texts in Math. 149, SpringerVerlag, New York 1994. Zbl 1106.51009 MR 1299730

[26] A. W. Reid, A note on trace-fields of Kleinian groups. Bull. London Math. Soc. 22 (1990), no. 4, 349-352. Zbl 0706.20038 MR 1058310

[27] E. B. Vinberg, Rings of definition of dense subgroups of semisimple linear groups. Izv. Akad. Nauk SSSR Ser. Mat. 35 (1971), 45-55 (in Russian). Zbl 0252.20043 MR 0279206 
[28] E. B. Vinberg, The smallest field of definition of a subgroup of the group PSL2. (Russian) Mat. Sb. 184 (1993), no. 10, 53-66; English transl. Russian Acad. Sci. Sb. Math. 80 (1995), no. 1, 179-190. Zbl 0831.20061 MR 1249413

[29] P. Will, Traces, cross-ratios and 2-generator subgroups of SU(2, 1). Canad. J. Math. 61 (2009), no. 6, 1407-1436. Zbl 1185.14038 MR 2588430

[30] B. Xie, The complex hyperbolic Kleinian groups with an invariant totally geodesic submanifold. Preprint, arXiv:1006.5581v2.

Received June 25, 2012; revised December 27, 2012

H. Cunha, Departamento de Matemática, Universidade Federal de Minhas Gerais, Belo Horizonte - MG, Brazil, 30123-970

E-mail: cunha@mat.ufmg.br

N. Gusevskii, Departamento de Matemática, Universidade Federal de Minhas Gerais, Belo Horizonte - MG, Brazil, 30123-970

E-mail: nikolay@mat.ufmg.br 